\title{
Normality and Its Variants on Fuzzy Isotone Spaces
}

\author{
Stephen M. Gathigi, Moses N. Gichuki, Paul A. Otieno, Hezron S. Were \\ Department of Mathematics, Egerton University, Egerton, Kenya \\ Email: machariastephen.y31@gmail.com, gichukih2002@yahoo.com, ptooex@yahoo.com, werehezron@gmail.com
}

Received August 7, 2013; revised September 8, 2013; accepted October 6, 2013

Copyright $(02013$ Stephen M. Gathigi et al. This is an open access article distributed under the Creative Commons Attribution License, which permits unrestricted use, distribution, and reproduction in any medium, provided the original work is properly cited.

\begin{abstract}
The study of fuzzy sets is specifically designed to mathematically represent uncertainty and vagueness by assigning values of membership to objects that belong to a particular set. This notion has been broadly extended to other areas of topology where various topological concepts have been shown to hold on fuzzy topology. Some notions naturally extend to closure spaces without requiring a lot of modification of the underlying topological ideas. This work investigates the variants of normality on fuzzy isotone spaces.
\end{abstract}

Keywords: Fuzzy Sets; Fuzzy Closure Space; Fuzzy Isotone Space; Fuzzy Normality

\section{Introduction}

The idea of a class of sets with a continuum of grade of membership, ranging between zero and one, was first introduced by Zadeh in 1965. A larger degree of membership of an object reflects a stronger sense of belonging to a set. If $A$ is a set in the ordinary sense of the term, then its membership takes only two values, 0 and 1 . The notions of inclusion, union, intersection, complement, relation and convexity can be extended to such sets [1].

Fuzzy closure spaces were introduced by [5] in an attempt to show that fuzzy topological spaces do not constitute a natural boundary for the validity of theorems and results. The axioms used to define fuzzy closure spaces are the modified Kuratowski closure axioms that have previously been used to extend the study of the concepts of topological spaces. The class of isotonic spaces is defined using only two Kuratowski closure axioms, namely the grounded axiom $(c(\varnothing)=\varnothing)$ and the isotone axiom

$$
(\forall A \subset B, c(A) \subset c(B))
$$

where $c: \mathcal{P}(X) \rightarrow \mathcal{P}(X)$ is the closure operator on a nonempty set $X$.

\section{Literature Review}

\subsection{Fuzzy Sets}

In [2], a fuzzy set in $X$ is defined as a function $\theta: X \rightarrow[0,1]$. Here $\theta(x)$ represents the degree of membership of $x \in X$ in the fuzzy set $\theta$.

\subsection{Crisp Fuzzy Sets}

Any subset $A$ of a set $X$ can be identified with its characteristic function $\chi_{A}: X \rightarrow\{0,1\}$ defined by;

$$
\chi_{A}(x)=\left\{\begin{array}{l}
1, \text { if } x \in A \\
0, \text { if } x \notin A
\end{array}\right.
$$

Such characteristic functions are fuzzy sets in $X$. Thus fuzzy sets generalize ordinary sets [3].

\subsection{Definitions on Fuzzy Sets}

Let $\mu$ and $\varphi$ be $[0,1]$-valued functions defined on a fixed set, i.e fuzzy sets on $X$. Then according to [3];

1) $\mu \leq \varphi$ implies $\mu(x) \leq \varphi(x)$ for every $x \in X$.

2) $\mu=\varphi$ implies $\mu(x)=\varphi(x)$ for every $x \in X$.

3) Maximum function:

$$
(\mu \vee \varphi)(x)=\max \{\mu(x), \varphi(x)\} .
$$

4) Minimum function:

$$
(\mu \wedge \varphi)(x)=\min \{\mu(x), \varphi(x)\} .
$$

5) Complement function: $(1-\mu)(x)=1-\mu(x)$. These are $[0,1]$-valued functions.

For two fuzzy sets $\mu$ and $\varphi$ in $X$;

1) $\mu$ and $\varphi$ are equal if and only if $\mu=\varphi$.

2) $\mu$ is contained in $\varphi$ if and only if $\mu \leq \varphi$.

3) The union of $\mu$ and $\varphi$ is $\mu \vee \varphi$.

4) The intersection of $\mu$ and $\varphi$ is $\mu \wedge \varphi$.

5) The complement of $\mu$ is $1-\mu$.

Let $\left\{\mu_{i}: i \in I\right\}$ be fuzzy sets. Then the union of 
$\left\{\mu_{i}: i \in I\right\}$ is defined by;

$$
\mathrm{V}_{i \in I} \mu_{i}(x)=\sup \left\{\mu_{i}(x): i \in I\right\}
$$

The intersection of $\left\{\mu_{i}: i \in I\right\}$ is defined by;

$$
\Lambda_{i \in I} \mu_{i}(x)=\inf \left\{\mu_{i}(x): i \in I\right\}
$$

If $\mu_{i^{\prime} s}$ are crisp, i.e. they are characteristic functions, then these suprema and infima are actually maxima and minima.

\subsection{Fuzzy Topology}

According to [4], a fuzzy topology on a set $X$ is a collection $\delta$ of fuzzy sets in $X$ satisfying

1) $0,1 \in \delta$, where 0 is equivalent to the empty set.

2) If $\mu$ and $\varphi$ belong to $\delta$, then $\mu \wedge \varphi=\delta$.

3) If $\left\{\mu_{i}: i \in I\right\}$ are fuzzy, then $\mathrm{V}_{i \in I} \mu_{i}=\delta$. The members of $\delta$ are called open fuzzy sets.

The pair $(X, \delta)$ is called a fuzzy topological space. Fuzzy sets of the form $1-\mu$, where $\mu$ is fuzzy are called closed fuzzy sets.

\subsection{Functions and Fuzzy Continuity}

Let $X$ and $Y$ be sets and $f: X \rightarrow Y$ be a function. For a fuzzy set $\mu$ in $Y$, the inverse image of $\mu$ under $f$ is the fuzzy set $f^{-1}(\mu)$ in $X$ defined by;

$$
f^{-1}(\mu)(x)=\mu(f(\mu))
$$

for $x \in X$. That is $f^{-1}(\mu)=\mu o f$.

For a fuzzy set $\mu$ in $X$, the image of $\mu$ under $f$ is the fuzzy set $f(\mu)$ in $Y$ defined for $y \in Y$ by;

$$
\begin{aligned}
& f(\mu)(y)= \\
& \begin{cases}\sup \left\{\mu(z): z \in f^{-1}(y)\right\} & \text { if } f^{-1}(y) \neq \varnothing \\
0 & \text { if } f^{-1}(y)=\varnothing\end{cases}
\end{aligned}
$$

\subsection{Fuzzy Continuity}

Given fuzzy topological spaces $(X, \delta)$ and $(X, \Phi)$, then according to [2], $f: X \rightarrow Y$ is fuzzy continuous if the inverse image under $f$ of any open fuzzy set $\mu$ in $Y$ is an open fuzzy set in $X$, i.e $f^{-1}(\mu) \in \delta$ whenever $\mu \in Y$.

The identity mapping $I d_{X}:(X, \delta) \rightarrow(X, \Phi)$ on a fuzzy topological space $(X, \delta)$ is fuzzy continuous.

\subsection{Closure and Interior Operation on Fuzzy Sets}

Let $(X, \delta)$ be a fuzzy topological space. The closure $\bar{\mu}$ and interior $\mu^{o}$ of a fuzzy set $\mu$ in $X$ are defined respectively by [3] as follows;

$$
\bar{\mu}=\inf \left\{\alpha: \alpha \geq \mu \text {, where } \mu^{c} \in \delta\right\}
$$

$$
\mu^{o}=\{\alpha: \alpha \leq \mu, \text { where } \mu \in \delta\} .
$$

It is easily seen that $\bar{\mu}$ is the smallest closed fuzzy set larger than $\mu$ and that $\mu^{o}$ is the largest open fuzzy set smaller than $\mu$. These definitions coincide with their analogous definitions on ordinary sets.

\subsection{Fuzzy Closure Spaces}

Let $I^{X}$ be the collection of all mappings from $X$ to the unit interval $[0,1]$, i.e $I^{X}$ is the collection of all fuzzy sets on the non-empty set $X$. Then from [5], an operator $c: I^{X} \rightarrow I^{X}$ is a fuzzy closure operator if and only if

1) $c(\alpha)=\alpha, \forall \alpha$ constant.

2) $c(\mu) \geq \mu$ for every $\mu \in I^{X}$.

3) $c(\mu) \vee c(\varphi)=c(\mu \vee \varphi)$ for every $\mu, \varphi \in I^{X}$.

4) $c(c(\mu))=c(\mu)$ for every $\mu \in I^{X}$.

The closure operator may also be used to characterize closed sets. A set $\mu$ is closed if $\mu=c(\mu)$. A fuzzy interior operator int: $I^{X} \rightarrow I^{X}$ is the dual of a closure operator. It is defined by;

1) $\operatorname{int}(\alpha)=\alpha, \forall \alpha$ constant.

2) $\operatorname{int}(\mu) \geq \mu$ for every $\mu \in I^{X}$.

3) $\operatorname{int}(\mu) \wedge c(\varphi)=c(\mu \wedge \varphi)$ for every $\mu, \varphi \in I^{X}$.

4) $\operatorname{int}(\operatorname{int}(\mu))=\operatorname{int}(\mu)$ for every $\mu \in I^{X}$.

Similarly, the interior operator may also be used to characterize open sets. A set $\mu$ is open if $\mu=\operatorname{int}(\mu)$.

A Cech fuzzy closure operator (or CF-closure operator) on a set $X$ is a function $c: I^{X} \rightarrow I^{X}$ satisfying the following three axioms;

1) $c(\alpha)=\alpha, \forall \alpha$ constant.

2) $c(\mu) \geq \mu$ for every $\mu \in I^{X}$.

3) $c(\mu) \vee c(\varphi)=c(\mu \vee \varphi)$ for every $\mu, \varphi \in I^{X}$.

The pair $(X, c)$ is called a fuzzy closure space or fcs. Clearly these axioms can easily be seen to be similar to the Kuratowski axioms in [6].

\section{Results}

The following are the main results of this work.

\subsection{Fuzzy Isotone Space}

A fuzzy isotone closure operator on a set $X$ is a function $c: I^{X} \rightarrow I^{X}$ satisfying the following two axioms;

1) $c(\alpha)=\alpha, \forall \alpha$ constant.

2) For every $\mu \leq \Phi, c(\mu) \leq c(\Phi)$.

The pair $(X, c)$ is called a fuzzy isotone space.

\subsection{Semi-Separated and Separated Fuzzy Sets}

We would like to modify the definitions of semi-sepa- 
rated and separated sets in order to have their equivalent characterization on fuzzy isotone spaces. This will facilitate the definition of complete normality on fuzzy isotone spaces.

In a fuzzy isotonespace $(X, c)$, two fuzzy subsets $\mu$ and $\Phi$ are called semi-separated if

$$
c(\mu) \wedge \Phi=\mu \wedge c(\Phi)=\varnothing .
$$

The fuzzy subsets $\mu$ and $\Phi$ are separated if there exists open fuzzy sets $U, V$ with $\mu \leq U$ and $\Phi \leq V$ such that $U \wedge V=\varnothing$. The openness of fuzzy sets on $X$ is defined using the dual of the closure operator, i.e the interior operator.

\section{Lemma}

Let $Y \leq X$. Then $\mu$ and $\Phi$ are semi-separated in $(X, c)$ if and only if $\mu$ and $\Phi$ are semi-separated in $\left(Y, c_{Y}\right)$, where $c_{Y}$ is the relativization of $c$ into $Y$.

\section{Proof}

Let $(X, c)$ be a fuzzy isotone space and $\mu, \Phi \in I^{Y}$ be semi-separated fuzzy sets in $(X, c)$. Then

$$
c(\mu) \wedge \Phi=\mu \wedge c(\Phi)=\varnothing .
$$

$$
\begin{aligned}
\text { But } & c_{Y}(\mu)=Y \wedge c(\mu) \text { hence } \\
& c_{Y}(\mu) \wedge \Phi=(Y \wedge c(\mu)) \wedge \Phi=Y \wedge \varnothing=\varnothing .
\end{aligned}
$$

Similarly, $c_{Y}(\Phi)=Y \wedge c(\Phi)$ hence

$$
\begin{aligned}
\mu \wedge c_{Y}(\Phi) & =\mu \wedge(Y \wedge c(\Phi)) \\
& =(\mu \wedge c(\Phi)) \wedge Y \\
& =\varnothing \wedge Y=\varnothing .
\end{aligned}
$$

Therefore, $\mu$ and $\Phi$ are semi-separated in $\left(Y, c_{Y}\right)$.

Conversely, let $\left(Y, c_{Y}\right)$ be a subspace of the fuzzy isotone space $(X, c)$ and $\mu$ and $\Phi$ are semi-separated in $\left(Y, c_{Y}\right)$. Of course $\mu, \Phi \in I^{X}$ and

$$
\begin{aligned}
& c_{Y}(\mu) \wedge \Phi=\mu \wedge c_{Y}(\Phi)=\varnothing . \\
& c_{Y}(\mu) \wedge \Phi=(Y \wedge c(\mu)) \wedge \Phi=c(\mu) \wedge \Phi=\varnothing .
\end{aligned}
$$

Similarly,

$$
\mu \wedge c_{Y}(\Phi)=\mu \wedge(Y \wedge c(\Phi))=\mu \wedge c(\Phi)=\varnothing
$$

Thus $\mu$ and $\Phi$ are semi-separated in $(X, c)$.

\subsection{Normality}

A fuzzy isotone space $(X, c)$ is normal if for every nonempty pair of fuzzy sets $A$ and $B$ in $I^{X}$ such that $c(A) \wedge c(B)=\varnothing$ there exists a fuzzyopen set $U$ such that $A \leq U$ and $c(U) \wedge B=\varnothing$.

Normality may be characterized via the existence of a fuzzy continuous real-valued function just as in topological spaces.
Let $(X, c)$ be a normal fuzzy isotone space. Then for each pair of disjoint fuzzy subsets $\mu$ and $\Phi$, there exists a fuzzy continuous function $f: X \rightarrow[0,1]$ such that $f \leq 0$ on $\mu$ and $f \leq 1$ on $\Phi$. Clearly, this characterization is analogous to the definition of normality via the existence of an Urysohn function on a normal topological space.

\subsection{Complete Normality}

A fuzzy isotone space $(X, c)$ is said to be completely normal if every fuzzy subspace of $X$ is normal.

\section{Theorem}

A fuzzy isotone space $(X, c)$ is completely normal if and only if for every pair $\mu$ and $\Phi$ of fuzzy subsets with $c(\mu) \wedge \Phi=\mu \wedge c(\Phi)=\varnothing$ then there exists disjoint fuzzy sets $U=\operatorname{int}(U) \geq \mu$ and $V=\operatorname{int}(V) \geq \Phi$.

\section{Proof}

Let $X$ be completely normal and $\mu, \Phi \in I^{X}$ be fuzzy sets with $c_{X}(\mu) \wedge \Phi=\mu \wedge c_{X}(\Phi)=\varnothing$. Denote $Y$, a subspace of $X$ by $Y=X-\left(c_{X}(\mu) \wedge c_{X}(\Phi)\right)$. Then $\mu, \Phi \in I^{Y}$ since

$$
\mu \wedge\left(c_{X}(\mu) \wedge c_{X}(\Phi)\right)=\left(\mu \wedge c_{X}(\Phi)\right) \wedge c_{X}(\mu)=\varnothing .
$$

Similarly,

$$
\Phi \wedge\left(c_{X}(\mu) \wedge c_{X}(\Phi)\right)=\left(c_{X}(\mu) \wedge \Phi\right) \wedge c_{X}(\Phi)=\varnothing .
$$

Clearly $c_{Y}(\mu)$ and $c_{Y}(\Phi)$ are closed sets in $Y$ such that $c_{Y}(\Phi) \wedge c_{Y}(\mu)=\varnothing$. Notice

$$
c_{Y}(\Phi) \wedge c_{Y}(\mu) \wedge Y \leq c_{X}(\mu) \wedge c_{X}(\Phi) \wedge Y .
$$

Therefore, since $X$ is completely normal, then $Y$ is normal and hence there exists $U$ and $V$ in $\tau(c)$ such that $c_{Y}(\mu) \leq U$ and $c_{Y}(\Phi) \leq V$.

Conversely, let $Y$ be a subspace of $X$ and $\mu, \Phi \in I^{X} \quad$ such that $\mu=c(\mu)$ and $\Phi=c(\Phi)$ with $\mu \wedge \Phi=\varnothing$. Then $\mu \wedge c_{Y}(\Phi)=\mu \wedge \Phi=\varnothing$. Similarly, $c_{Y}(\mu) \wedge \Phi=\mu \wedge \Phi=\varnothing$. Therefore, by the hypothesis of the theorem, there exists disjoint fuzzy sets

$$
U=\operatorname{int}(U) \geq \mu \text { and } V=\operatorname{int}(V) \geq \Phi .
$$

The fuzzy sets $U \wedge Y$ and $V \wedge Y$ are disjoint and contained in $I^{Y} U \wedge Y \geq \mu$ and $V \wedge Y \geq \Phi$. Hence $Y$ is normal and $X$ is therefore completely normal.

\subsection{Perfect Normality}

Perfect normality has not been defined in fuzzy closure spaces. Therefore, different characterizations are given under this section as modifications from topological spaces. A few basic concepts have to be carried over from general topological spaces before any meaningful definition of perfectly normal isotonic spaces can be 
given.

\subsubsection{Preliminary Definitions}

It is known form point-set topology and from fuzzy topology that though the countable union of closed sets need not be closed, and the countable intersection of open sets need not be open, such sets occur frequently in analysis. The occurrence of such sets guarantees perfect normality on a space $X$.

A fuzzy set $G$ is called a $G_{\delta}$-set if and only if

$$
G=\bigwedge_{i=1}^{\infty} \mu_{i}=\inf \left\{\mu_{i}(x): i \in I\right\},
$$

where $\mu_{i}=\operatorname{int}\left(\mu_{i}\right) \forall i$. A set $F$ is called an $F_{\sigma}$-set if

$$
F=\mathrm{V}_{i=1}^{\infty} \beta_{i}=\sup \left\{\beta_{i}(x): i \in I\right\}
$$

where $\beta_{i}=c\left(\beta_{i}\right) \forall i$.

A fuzzy isotone space $(X, c)$ is perfectly normal if $X$ is normal and for every fuzzy subset $\mu=\operatorname{cl}(\mu)$ of $X, \mu$ is a $G_{\delta}$-set. That is for every closed fuzzy set $\mu$ in $X, \mu=\Lambda_{i=1}^{\infty} M_{i}, M_{i}=\operatorname{int}\left(M_{i}\right) \forall i$. Equivalently, a normal isotonic space $(X, c l)$ is perfectly normal if every open fuzysubset of $X$ is an $F_{\sigma}$-set. That is, for every fuzzy set $\Phi=\operatorname{int}(\Phi)$, then

$$
\Phi=\bigvee_{i=1}^{\infty} N_{i}, N_{i}=\operatorname{cl}\left(N_{i}\right) \forall i .
$$

\subsubsection{Theorem}

The fuzzy isotone space $(X, c)$ is perfectly normal if for every $A, B \in I^{X}$ such that $A=c(A), B=c(B)$ and $c(A) \cap c(B)=\varnothing$, then $\exists$ a fuzzy continuous function $f: X \rightarrow[0,1]$ that precisely separates $A$ and $B$. That is $f^{-1}\{0\}=A$ and $f^{-1}\{1\}=B$

\subsubsection{Theorem}

Every perfectly normal fuzzy isotone space $(X, c)$ is completely normal.

\section{Proof}

Since a fuzzy isotone space is completely normal if and only if every subspace is normal, then in order to show that perfect normality implies complete normality, it suffices to show the heredity of perfect normality. Let $(X, c)$ be perfectly normal fuzzy isotone space. Then for every closed fuzzy set $\mu \in I^{X}$ there exists a fuzzy continuous function $f: X \rightarrow[0,1]$ such that $f^{-1}\{0\}=\mu$. Let $Y \leq X$ be a subspace of $(X, c)$ and $\mu \leq I^{Y}$ be closed. Then there exists a fuzzy closed set $\mu_{0} \in I^{X}$ such that $\mu=\mu_{0} \wedge Y$. Since $\mu_{0}$ is closed in $X$, then there exists a fuzzy continuous function $f: X \rightarrow[0,1]$ such that $\mu_{0}=f^{-1}\{0\}$. But $g=\left.f\right|_{Y}: Y \rightarrow[0,1]$ is also fuzzy continuous and $g^{-1}\{0\}=f^{-1}\{0\} \wedge Y=\mu_{0} \wedge Y=\mu$. Therefore $Y$ is perfectly normal and hence normal. This implies that $X$ is completely normal and hence perfect normality implies complete normality.

\section{Conclusion}

The variants of normality naturally extend to the class of fuzzy isotone spaces and to the fuzzy closure spaces generally. Therefore, on fuzzy isotone spaces, perfect normality implies complete normality which implies normality.

\section{REFERENCES}

[1] L. A. Zadeh, "Fuzzy Sets," Information and Control, Vol. 8, No. 3, 1965, pp. 338-353. http://dx.doi.org/10.1016/S0019-9958(65)90241-X

[2] C. L. Chang, "Fuzzy Topological Spaces," Journal of Mathematical Analysis and Applications, Vol. 24, No. 1, 1968, pp. 265-270. http://dx.doi.org/10.1016/0022-247X(68)90057-7

[3] R. Lowen, "Fuzzy Topological Spaces and Fuzzy Compactness," Journal of Mathematical Analysis and Applications, Vol. 56, No. 3, 1976, pp. 621-633. http://dx.doi.org/10.1016/0022-247X(76)90029-9

[4] T. E. Gantner, R. C. Steinlage and H. R. Warren, "Compactness in Fuzzy Topological Spaces," Journal of Mathematical Analysis and Applications, Vol. 62, No. 3, 1978, pp. 547-562. http://dx.doi.org/10.1016/0022-247X(78)90148-8

[5] A. S. Mashhour andM. H. Ghanim, "Fuzzy Closure Spaces," Journal of Mathematical Analysis and Applications, Vol. 106, No. 3, 1985, pp. 154-170. http://dx.doi.org/10.1016/0022-247X(85)90138-6

[6] W. J. Thron, "What Results Are Valid on Cech-Closure Spaces," Topology Proceedings, Vol. 6, 1981, pp. 135158. 